\title{
Eficacia del programa educativo "Mi sol" para la prevención del abuso sexual infantil en menores de edad de la ciudad de Juliaca, Perú
}

\section{Efficacy of the educational program "Mi sol" for the prevention of child sexual abuse in children in the city of Juliaca, Peru}

\author{
Oscar Mamani-Benito ${ }^{11}$, Jhon Pinto-Cahuapaza ${ }^{2}$, Jubitsa Núñez-Vargas ${ }^{3}$, \\ Flavio Quispe Chañi ${ }^{4}$, Sheila Mamani Ordoño ${ }^{5}$, Rosa Vargas Huamantuco ${ }^{6}$ \\ Universidad Peruana Unión, Perú1234567
}

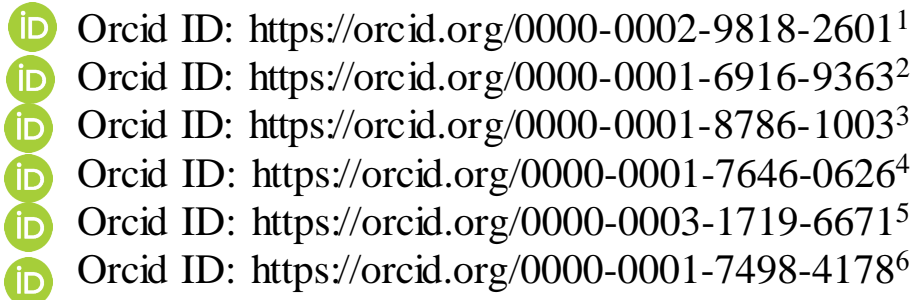

Recibido: 30 de noviembre de 2019

Aceptado: 12 de febrero de 2020

\begin{abstract}
Resumen
El presente estudio tuvo el objetivo de determinar la eficacia del programa "Mi Sol" en la prevención del abuso sexual infantil (ASI) a través del desarrollo de conocimientos al respecto y habilidades de prevención. El estudio corresponde a un diseño cuasi experimental, realizado sobre un grupo de 28 estudiantes de nivel primario de una institución educativa estatal de la ciudad de Juliaca. Así también, se contó con la participación de un grupo de control conformado por 20 estudiantes. Se aplicó el programa denominado "Mi Sol" elaborado por UNICEF-Chile, y para probar su eficacia se aplicó un cuestionario de conocimientos sobre ASI y habilidades de prevención, los mismos que demostraron ser válidos y confiables. Los resultados en cuanto a la variable "Conocimientos sobre ASI" antes de la intervención demostraron que el puntaje de la media fue de 22.89 y después 28.32 ( $\mathrm{p}=.009$ ); en cambio, el grupo control-antes de la intervención-arrojó un resultado de 22.40 y luego de $22.45(\mathrm{p}=.241)$. Por otra parte, en cuestión de las habilidades de prevención, en el grupo experimental la media antes de la
\end{abstract}

${ }^{1}$ Correspondencia al autor

E-mail: oscar.mb@upeu.edu.pe 
intervención fue de 19.14 y después $22.14(\mathrm{p}=.024)$; en cambio, en el grupo control antes fue de 16.95 y después $16.80(\mathrm{p}=.186)$. Se concluye que el programa "Mi Sol" es eficaz para menores de edad, pues incrementa conocimientos sobre ASI y desarrolla habilidades de prevención, con el objetivo de prevenir situaciones de riesgo que conlleva ser víctima de abuso sexual.

Palabras claves: abuso sexual, prevención primaria, niñez, maltrato infantil, familia

\begin{abstract}
This article aimed to determine the effectiveness of the "Mi Sol" program in the prevention of child sexual abuse (ASI, in spanish) through the development of knowledge and prevention skills about it. The study corresponds to a quasi-experimental design, carried out on a group of 28 primary level students from a state educational institution in the city of Juliaca, Perú. Likewise, there was the participation of a control group made up of 20 students. The program called "Mi Sol" developed by UNICEF-Chile was applied, and to test its effectiveness, a questionnaire of knowledge about ASI and prevention skills was applied, which proved to be valid and reliable. The results regarding the variable "Knowledge about ASI" before the intervention showed that the mean score was 22.89 and then $28.32(\mathrm{p}=.009)$; however, the control group - before the intervention - yielded a result of 22.40 and after 22.45 ( $\mathrm{p}=.241$ ). On the other hand, regarding prevention skills, in the experimental group the mean before the intervention was 19.14 and after $22.14(\mathrm{p}=.024)$; on the other hand, in the control group it was before 16.95 and after $16.80(\mathrm{p}=.186)$. It is concluded that the "Mi Sol" program is effective for minors, since it increases knowledge about ASI and develops prevention skills, with the aim of preventing risk situations that come with being a victim of sexual abuse.
\end{abstract}

Keywords: sexual abuse, primary prevention, childhood, child abuse, family

\title{
Introducción
}

El abuso sexual infantil (ASI) reúne una serie de conductas de connotación sexual realizada en contra de niños y niñas. De entre todas estas prácticas, son los tocamientos corporales o la misma penetración las más perjudiciales (Arredondo, Saavedra y Troncoso, 2016). En este caso, Acuña (2014) menciona que el ASI, como problemática, es conocido desde décadas y en la actualidad se ha convertido en un tema de preocupación a nivel mundial, que además es considerado como una transgresión de los derechos humanos más fundamentales, siendo este uno de los métodos de maltrato que genera mayores traumas con consecuencias a corto y largo plazo, tanto para la familia, la sociedad y las mismas víctimas.

Según la Organización Mundial de la Salud (OMS, 2016), los niños y adolescentes menores de 14 años son los más vulnerables a sufrir este tipo de violencia en manos de familiares y personas cercanas. Al respecto, la misma OMS revela que mundialmente existen 73 millones de niños y 150 millones de niñas menores de 18 años que sufren violencia sexual en forma de 
tocamientos y relaciones sexuales forzadas; en consecuencia, la prevalencia mundial de víctimas sexuales en la niñez fue de $27 \%$ en niñas y $14 \%$ en niños. En América Latina, investigadores de la Universidad Autónoma de México encontraron que el 77\% de la víctimas del ASI son niñas de un promedio de 5 a 7 años de edad, siendo los agresores los mismos familiares y personajes más cercanos de la víctima (padre, hermano, cuñado, primo, abuelo, padrastro, tío, amigos de la propia familia, etc.; Velázquez, Delgadillo y González, 2013).

En el Perú, según el Instituto Nacional de Estadística e Informática (INEI, 2018), el 64.2\% de mujeres menores de 18 años fueron violentadas sexualmente. Esta cifra fue registrada por la Policía Nacional del Perú (PNP) entre enero y junio del año 2018. Al respecto, la literatura científica revela que hay factores de riesgo que influyen en el ASI por parte de los agresores; estos han sido señalados en múltiples estudios como pertenecientes a una pandilla, con consumo perjudicial y/o ilícito de alcohol o drogas, personalidad antisocial, exposición en la niñez a la violencia entre los padres, antecedentes de abuso físico o sexual en la niñez, escasa educación, promiscuidad sexual o infidelidad, opiniones favorables a la inequidad de género, entre otros (Redondo, Pérez y Martínez, 2014).

Teniendo en cuenta todas estas aproximaciones, no es nada nuevo valorar los daños graves a futuro en las victimas; en este caso, diversos estudios han demostrado que ser víctima de ASI altera el desarrollo neurológico en el sistema nervioso central, ya que cada estructura del cerebro del niño sufre una alteración negativa que impacta su desarrollo natural. Posteriormente, esta situación puede desencadenar una experiencia donde las victimas presentan mayor riesgo de desarrollar enfermedades mentales, como es el caso de un trastorno de estrés postraumático (Acuña, 2014). Por su parte. Rodríguez (2012) menciona que la mayoría de las víctimas de ASI presentan trastornos depresivos y bipolares, síntomas y trastornos de ansiedad, trastorno límite de la personalidad, negligencia en las obligaciones, ausencia de autoprotección, conductas autolesivas, ideas suicidas y baja autoestima. Además de la aparición de insomnio, pérdida y aumento de apetito, dolores de cabeza, y debilidad muscular, surgen conductas como: depresión, ansiedad, aplanamiento afectivo, miedo, vergüenza, culpa, enojo, distracción, distorsión del tiempo, bajo rendimiento escolar, pensamientos en el evento, pesadillas, distanciamiento familiar y social y constante hipervigilancia (Velázquez et al., 2013).

En este contexto, y tomando en cuenta que en el Perú hace faltan estrategias y estudios preventivos-específicamente en la ciudad de Juliaca donde el índice de casos incrementa a 
través del tiempo (La República, 2019)—-la presente investigación tiene el objetivo de determinar si el programa educativo "Mi Sol" es eficaz para prevenir el abuso sexual infantil a través del desarrollo de conocimientos sobre ASI y habilidades de prevención en menores de edad de una institución educativa estatal de nivel primario.

\section{Materiales y método}

\section{Diseño y población}

Esta investigación comprende un diseño cuasi experimental, con evaluación pre y post intervención. Se contó con la participación de 48 estudiantes de ambos sexos pertenecientes al quinto grado de educación primaria, de una institución educativa pública de la ciudad de Juliaca, Perú; esta población no manifiesta posibles condiciones físicas o psicológicas que imposibilite su participación en la investigación. Esta fue distribuida en dos grupos: experimental (28) y control (20). La configuración de ambos grupos estuvo sujeta a criterios de inclusión y exclusión.

\section{Instrumentos}

En la recolección de información sobre características demográficas como edad, sexo y religión, se utilizó un cuestionario demográfico; en cambio, para la variable "Conocimientos sobre ASI" y la variable "Habilidades de prevención," se recurrió a un cuestionario elaborado por Del Campo y López (2006) en un estudio similar. Su composición denota la existencia de 35 ítems, distribuidos para cada variable, en este caso "Conocimientos sobre ASI" (2. 3. 4. 5. 7. 9. 10. 11. 12. 14. 15. 16. 17. 19. 22. 24. 27. 28. 33 y 35) y "Habilidades de prevención" (1. 6. 8. 13. 20. 21.23. 25. 26. 29. 30.31. 32 y 34).

En cuanto a sus propiedades psicométricas, se procedió a validar el instrumento mediante el juicio de tres expertos, quienes valoraron el contenido de la prueba a través de criterios como: claridad de redacción, coherencia, pertinencia y congruencia con el constructo. Producto de ello, se obtuvo un índice de acuerdo de .90 para conocimientos y .987 para habilidades. De igual modo, la confiabilidad fue hallada mediante el coeficiente Alfa de Cronbach. En esta caso, el valor obtenido para conocimientos fue de .89 y para habilidades de .84 .

\section{Programa}

La elaboración de esta metodología de trabajo corresponde al denominado "Mi Sol," creado en Chile por el Ministerio de Educación y el Fondo de las Naciones Unidas Para la Infancia (UNICEF, 2012). En cuanto a su contenido, este se divide de la siguiente manera: 
- Módulo 1. Actividades dirigidas a los padres de familia.

$\checkmark$ Sesión $\mathrm{N}^{\circ} 1 \mathrm{El}$ compromiso de la familia en la intervención del abuso sexual infantil.

$\checkmark$ Sesión N² La familia en la prevención del abuso sexual infantil

$\checkmark$ Sesión N³ Consecuencias del abuso sexual.

- Módulo 2. Actividades dirigidas a estudiantes del $5^{\circ}$ grado de primaria.

$\checkmark$ Sesión $N^{\circ} 1$ Yo me expreso, tú te expresas.

$\checkmark$ Sesión $\mathrm{N}^{\circ} 2 \mathrm{Mi}$ cuerpo, mi sexualidad.

$\checkmark$ Sesión $N^{\circ} 3$ Cariños buenos, cariños malos.

$\checkmark$ Sesión $\mathrm{N}^{\circ} 4$ Secretos buenos, secretos malos.

$\checkmark$ Sesión $N^{\circ} 5$ Yo confió en mí, yo confío en mi ronda.

$\checkmark$ Sesión $N^{\circ} 6$ Ojo con la web.

\section{Procedimientos}

La intervención fue llevada a cabo entre los meses de mayo a junio del año 2019, en tres fases: pretest, intervención y postest. Se aplicó el pretest con el fin de recolectar datos sociodemográficos e identificar el nivel de conocimientos sobre ASI y habilidades de prevención, mediante la técnica de la encuesta. En la segunda fase se aplicó el programa educativo, que tuvo un alcance de seis sesiones para los menores de edad y tres para los padres. Cada sesión tuvo una duración de entre 45 a 90 minutos aplicando una metodología participativa e involucrando talleres vivenciales. Finalmente, se aplicó un postest para contrastar los indicadores iniciales con los obtenido luego de la intervención.

\section{Análisis estadístico}

Se utilizó el programa SPSS versión 22.0. Los resultados descriptivos se expresaron en tablas de frecuencia y porcentaje. Para el análisis inferencial se consideró un nivel de significancia $\leq 0.05$. El estadístico de prueba fue elegido en razón de la prueba de normalidad, específicamente Shapiro-Wilk $(\mathrm{N}<30)$, con lo cual se logró determinar cómo apropiado una prueba paramétrica, en este caso $t$ de student, debido a que los datos en ambos grupos demostraron seguir una distribución normal.

\section{Consideraciones éticas}


Este estudio respeta la ética en la investigación. Evidencia de ello es que se procedió a gestionar la autorización por parte del centro educativo, y se obtuvo el consentimiento informado de los padres de familia y el asentimiento de los participantes del grupo experimental y grupo de control.

\section{Resultados}

Las características del grupo experimental (tabla 1) revelan que el $82.1 \%$ de los participantes tienen 10 años y el 17.9\%, 11 años. El 57.1\% son varones y el 42.9 mujeres. El $75 \%$ vive con ambos padres: $10.7 \%$ con mamá, $7.1 \%$ con papá y $7.1 \%$ con otros familiares. En cuanto a la filiación religiosa, el $57.1 \%$ se consideran católicos, $10.7 \%$ adventistas, $7.1 \%$ evangélicos y 25\% de otras denominaciones. En cuanto al grupo de control (tabla 1), 65\% tienen 11 años y 35\%, 10 años; el 70\% son mujeres y 30\% varones. Por otro lado, el 60\% vive con ambos padres, $30 \%$ solo con mamá, $10 \%$ únicamente con papá y $0 \%$ con otros familiares. Por último, el $75 \%$ de este grupo se considera católico, 20\% adventista, 5\% evangélico y $0 \%$ de otra denominación.

Tabla 1

Características demográficas del grupo experimental y control

\begin{tabular}{llcccc}
\hline \multirow{2}{*}{ Variables } & & \multicolumn{2}{c}{ Grupo experimental } & \multicolumn{2}{c}{ Grupo control } \\
\cline { 3 - 6 } & & $\mathrm{F}$ & $\%$ & $\mathrm{f}$ & $\%$ \\
\hline Edad & 10 años & 23 & 82.1 & 7 & 35.0 \\
\multirow{3}{*}{ Sexo } & 11 años & 5 & 17.9 & 13 & 65.0 \\
& Masculino & 16 & 57.1 & 6 & 30.0 \\
& Femenino & 12 & 42.9 & 14 & 70.0 \\
& Ambos padres & 21 & 75 & 12 & 60.0 \\
\multirow{3}{*}{ Religión } & Mamá & 3 & 10.7 & 6 & 30.0 \\
& Papá & 2 & 7.1 & 2 & 10.0 \\
& Otros & 2 & 7.1 & 0 & 0 \\
& Católico & 16 & 57.1 & 15 & 75.0 \\
& Adventista & 3 & 10.7 & 4 & 20.0 \\
& Evangélico & 2 & 7.1 & 1 & 5.0 \\
\hline
\end{tabular}

En la tabla 2 se reportan las frecuencias y porcentajes según los niveles de los conocimientos sobre ASI y habilidades de prevención antes y después de la intervención en ambos grupos. En el grupo experimental y en cuanto a la primera variable-antes de la intervención, el $10.7 \%$ evidenció un nivel bajo, 39.3\% un nivel medio y 50\% un nivel alto. 
Luego de la intervención, ningún participante demostró un nivel bajo; decreció a 35.7\% quienes tenían un nivel medio e incrementó a $64.3 \%$ quienes demostraban un nivel alto. El mismo análisis en el grupo de control revela que, antes de la intervención, el 15\% demostraba un nivel bajo, 55\% un nivel medio y $30 \%$ un nivel alto. Luego de la intervención, decreció a 10\% quienes evidenciaban un nivel bajo, incrementó a $65 \%$ quienes demostraban un nivel medio y disminuyó a 35\% quienes tenían un nivel alto.

En cuanto a la variable "Habilidades de prevención" en el grupo experimental, la misma tabla 2 indica que, antes de la intervención, un 7.1\% demostraba un nivel bajo, $21.4 \%$ un nivel medio y $71.4 \%$ un nivel alto. Después de aplicar el programa, disminuyó a 3.6\% quienes tenían un nivel bajo, disminuyó a 17.9\% quienes tenían nivel medio e incrementó a $78.6 \%$ quienes demostraban un nivel alto. El mismo análisis en el grupo de control da cuenta que, antes de intervenir, el 5\% evidenciaba un nivel bajo, 50\% un nivel medio y $45 \%$ un nivel alto. Luego de intervenir se mantuvo un 5\% con nivel bajo, disminuyó a $45 \%$ quienes tenían un nivel medio e incrementó a 50\% los del nivel alto.

Tabla 2

Niveles de conocimiento sobre ASI y habilidades de prevención antes y después de la intervención en ambos grupos

\begin{tabular}{lllcccccccc}
\hline & & \multicolumn{4}{c}{ Grupo experimental } & \multicolumn{3}{c}{ Grupo control } \\
\cline { 3 - 11 } & & & Bajo & Medio & Alto & Total & Bajo & Medio & Alto & Total \\
\hline Conocimientos & Pre test & $\mathrm{f}$ & 3 & 11 & 14 & 28 & 3 & 11 & 6 & 20 \\
sobre ASI & & $\%$ & 10.7 & 39.3 & 50.0 & 100.0 & 15.0 & 55.0 & 30.0 & 100.0 \\
& Pos test & $\mathrm{f}$ & 0 & 10 & 18 & 28 & 2 & 13 & 5 & 20 \\
& & $\%$ & 0.0 & 35.7 & 64.3 & 100.0 & 10.0 & 65.0 & 25.0 & 100.0 \\
Habilidades de & Pre test & $\mathrm{f}$ & 2 & 6 & 20 & 28 & 1 & 10 & 9 & 20 \\
prevención & & $\%$ & 7.1 & 21.4 & 71.4 & 100.0 & 5.0 & 50.0 & 45.0 & 100.0 \\
& \multirow{2}{*}{ Pos test } & $\mathrm{f}$ & 1 & 5 & 22 & 28 & 1 & 9 & 10 & 20 \\
& & $\%$ & 3.6 & 17.9 & 78.6 & 100.0 & 5.0 & 45.0 & 50.0 & 100.0 \\
\hline
\end{tabular}

Las diferencias porcentuales antes discutidas en ambos grupos pueden ser aclarados en la tabla 3. En cuanto a la variable "Conocimientos sobre ASI" en el grupo experimental, el puntaje de la media antes de intervenir fue de 22.89 y después 28.32, lo cual según el estadístico de prueba es significativo $(\mathrm{p}=.009)$. En cambio, en el grupo de control, el puntaje de la media antes de intervenir fue de 22.40 y después 22.45 , que según el estadístico de prueba no es significativo $(\mathrm{p}=.841)$. Por otra parte, en cuanto a la variable "Habilidades de prevención" en el grupo experimental (ver tabla 3), se observa que el puntaje de la media antes de intervenir fue de 19.14 y después 22.14; esta diferencia según el estadístico de prueba es significativa ( $p$ 
$=.024)$. En cambio, en el grupo de control, el puntaje de la media antes de intervenir fue de 16.95 y después 16.80 , y según el estadístico de prueba esta diferencia no es significativa $(\mathrm{p}=$ $.186)$.

Tabla 3

Estadísticos de prueba para determinar diferencias significativas antes y después de la intervención en ambos grupos

\begin{tabular}{|c|c|c|c|c|c|c|c|c|}
\hline \multirow{3}{*}{\begin{tabular}{l}
\multicolumn{1}{c}{ Variable } \\
Conocimientos \\
sobre ASI
\end{tabular}} & \multicolumn{2}{|c|}{ Grupos } & \multirow{2}{*}{$\frac{N}{28}$} & \multirow{2}{*}{$\frac{\text { Media }}{22.89}$} & \multirow{2}{*}{$\begin{array}{c}\text { DS } \\
9.93\end{array}$} & \multirow{3}{*}{$\frac{\mathrm{s}-\mathrm{W}}{.073}$} & \multirow{3}{*}{$\frac{t}{-3.70}$} & \multirow{3}{*}{$\frac{\mathrm{p}}{.009}$} \\
\hline & \multirow[t]{2}{*}{ Experimental } & Pre-test & & & & & & \\
\hline & & Post-test & 28 & 28.32 & 6.15 & & & \\
\hline \multirow{6}{*}{$\begin{array}{l}\text { Habilidades de } \\
\text { prevención }\end{array}$} & \multirow[t]{2}{*}{ Control } & Pre-test & 20 & 22.40 & 6.10 & \multirow{2}{*}{.342} & \multirow{2}{*}{-.201} & \multirow{2}{*}{.841} \\
\hline & & Post-test & 20 & 22.45 & 6.18 & & & \\
\hline & Experimental & Pre-test & 28 & 19.14 & 5.81 & \multirow{2}{*}{.121} & \multirow{2}{*}{-2.38} & \multirow{2}{*}{.024} \\
\hline & & Post-test & 28 & 22.14 & 5.87 & & & \\
\hline & \multirow[t]{2}{*}{ Control } & Pre-test & 20 & 16.95 & 4.53 & \multirow{2}{*}{.092} & \multirow{2}{*}{1.37} & \multirow{2}{*}{.186} \\
\hline & & Post-test & 20 & 16.80 & 4.43 & & & \\
\hline
\end{tabular}

\section{Discusión}

Los principales resultados dan cuenta de la efectividad del programa educativo "Mi Sol" en el desarrollo de conocimientos sobre abuso sexual infantil (ASI) y habilidades de prevención en menores de edad; en consecuencia, se confirma la posibilidad de prevenir el abuso sexual en niños. Este importante hallazgo corrobora el impacto positivo del programa "Mi Sol," que justamente es un manual con orientaciones para la prevención del abuso sexual infantil desde la comunidad educativa.

Resultados similares en el ámbito nacional fueron encontrados por Álvarez-Apaza, Machaca-Mamani y Mamani-Benito (2019), quienes aplicaron el programa en el departamento de Puno, tomando como población a menores de edad del nivel primario de una institución educativa estatal de la ciudad de Puno. Los resultados de tal investigación concluyen haber incrementado factores protectores como son los conocimientos y habilidades; en este caso, los autores unificaron ambas variables proponiendo un baremo total; en cambio, en la presente investigación, se respetó el diseño original del instrumento propuesto por Del Campo y López (2006) haciendo un análisis para cada variable. Más allá de las diferencias metodológicas, debe señalarse que en la región Puno ambos resultados demuestra la importancia que cumplen los programas educativos orientados a la prevención en poblaciones vulnerables. Así también, los hallazgos aquí encontrados pueden ser contrastados con los resultados de otro programa de intervención aplicado a nivel internacional por Del Campo y López (2006) quienes intervinieron-aunque no con el programa "Mi Sol"-sobre menores de edad de entre 8 a 12 
años, obteniendo al igual que la presente un impacto muy positivo sumando conocimientos de sobre este riesgo y mejorando sus habilidades de afrontamiento ante un posible abuso sexual.

En tal sentido, es de suma importancia aplicar este tipo de programas en centros educativos en el departamento de Puno. El hecho de que los estudiantes conozcan sobre este problema los protege y advierte de futuras situaciones de peligro. Al respecto, Deza (2005) ya manifestaba hace muchos años la importancia de reducir los factores de riesgo a través de incrementar el grado de conocimiento que un niño posee sobre el abuso sexual infantil. Según el autor, el contenido de los programas debe priorizar temas como la naturaleza del abuso sexual, propiedad sobre su cuerpo, sistemas de apoyo y culpabilidad. Aunque puede parecer contraproducente hablar de este tema con menores de edad, estudios como el realizado por Viñas et al. (2015) y Çeçen-Eroğul y Kaf (2013) demostraron que los niños desarrollan una actitud positiva ante la implantación de un programa de incremento de conocimientos sobre sexualidad saludable, lo cual ayuda a la detección de padecer un abuso sexual.

Por otra parte, es importante trabajar las habilidades de prevención, que algunos autores denominan "habilidades de afrontamiento" y son considerados un factor protector (Deza, 2005; Vicente, 2017); esto, si se reconoce que el tener un mero conocimiento de lo que implica un abuso sexual no es suficiente para no sufrir algún tipo de violencia. El(la) niño(a) necesita adiestrarse en estrategias que, según Quiroz (2015), solo se pueden desarrollar mediante programas que cambien la predisposición de los niños y les brinde herramientas de autoprotección ante situaciones de riesgo. Esta competencia forma un aspecto ampliamente desarrollado en el programa "Mi Sol."

Otro punto clave para lograr la eficacia del programa tiene que ver con el trabajo realizado con los padres de familia, pues las evidencias indican que cerca del $50 \%$ de ellos no dialoga con sus hijos acerca de este tema (Morillo, Montero \& Colmenares, 2012). En este caso, el programa "Mi Sol" cuenta con tres sesiones para concientizar sobre el rol de la familia como principal agente protector ante una situación de riesgo, incluso ya cometido el delito.

Una de las principales limitaciones que revela esta investigación, tiene que ver con el hecho de que no se ha logrado medir los aprendizajes en el tiempo, tanto en conocimientos como habilidades. En tal sentido, otros estudios (como el realizado por Czerwiski et al, 2018) demostraron que programas similares tuvieron un impacto en el aprendizaje a largo plazo, durante un periodo de tres meses después de haber ejecutado la última sesión. Así también, Bustamante et al. (2019) obtuvo un resultado similar, demostrando que el aprendizaje obtenido 
era vigente aun seis meses después de la última aplicación. Por lo tanto, se requiere que futuras investigaciones involucren mediciones en el tiempo a fin de confirmar la continuidad de los aprendizajes y estrategias logrados en el tiempo.

Finalmente, una de las fortalezas de esta investigación tiene que ver con el valor de los hallazgos antes discutidos, ya que estos pueden servir para que la comunidad científica impulse la creación de políticas educativas que permitan empoderar a menores de edad frente a situaciones de vulnerabilidad (Sánchez. 2015). Por lo tanto, se concluye que el programa educativo "Mi Sol" es eficaz en el desarrollo de conocimientos sobre abuso sexual infantil, y en el incremento de habilidades de prevención del mismo. Esto implica que los(as) niños(as) sometidos a la intervención logren empoderarse y puedan afrontar situaciones de riesgo defendiéndose de agresores que quieran aprovecharse de su fragilidad.

\section{Referencias}

Álvarez-Apaza, R. A., Machaca-Mamani, A., y Mamani-Benito, O. J. (2019). Eficacia de un programa psicoeducativo para prevenir el abuso sexual en menores de educación primaria. Casus, 4(2), 54-61. Recuperado de https://casus.ucss.edu.pe/index.php/casus/article/view/162/125

Acuña Navas, M. J. (2014). Abuso sexual en menores de edad: generalidades. consecuencias y prevención. Medicina legal de Costa Rica, 31(1), 57-69. Recuperado de https://www.scielo.sa.cr/pdf/mlcr/v31n1/art06v31n1.pdf

Arredondo, V., Saavedra, C., y Troncoso, C. (2016). Develación del abuso sexual en niños y niñas atendidos en la Corporación Paicabi. Revista latinoamericana de ciencias sociales. Niñez y juventud, 14(1), 385-399. Recuperado de http://www.scielo.org.co/pdf/rlcs/v14n1/v14n1a27.pdf

Bustamante, G., Andrade, M. S., Mikesell, C., Cullen, C., Endara, P., Burneo, V., Yepéz, P., Ávila, S., Ponce, P,. y Grunauer, P. (2019). "I have the right to feel safe": Evaluation of a school-based child sexual abuse prevention program in Ecuador. Child abuse \& neglect, 91(1), 31-40. doi.org/10.1016/j.chiabu.2019.02.009

Çeçen-Eroğul, A. R., \& Kaf, Ö. (2013). The effectiveness of psycho-educational school-based child sexual abuse prevention training program on turkish elementary students.

Educational sciences: Theory \& practice, 13(2), 725-729. Recuperado de https://files.eric.ed.gov/fulltext/EJ1017300.pdf 
Czerwinski, F., Finne, E., Alfes, J., y Kolip, P. (2018). Effectiveness of a school based intervention to prevent child sexual abuse-evaluation of the german IGEL program. Child abuse \& neglect, 86(4), 109-122. doi.org/10.1016/j.chiabu.2018.08.023.

Del Campo, A., y López, F. (2006). Evaluación de un programa de prevención de abusos sexuales a menores en educación primaria. Psicothema, 18(1), 1-8. Recuperado de http://www.psicothema.com/pdf/3168.pdf

Deza, S. (2005). Factores protectores en la prevención del abuso sexual infantil. Liberabit, 11(11), 210-21. Recuperado de http://pepsic.bvsalud.org/scielo.php?script=sci_arttext\&pid=S1729-48272005000100003

Iglesias, M., y Ibieta, B. (2012). Orientación para la prevención del abuso sexual desde la comunidad educativa. Baja California, México: Secretaría de Educación Pública.

Recuperado de http://convivenciaescolar.mineduc.cl/wpcontent/uploads/2019/04/Orientaciones-para-la-prevenci\%C3\%B3n-del-abuso-sexualinfantil-desde-la-comunidad-educativa.pdf

Instituto Nacional de Estadística e Informática (2018). Perú: Indicadores de violencia familiar y sexual. Lima, Perú. Recuperado de https://www.inei.gob.pe/media/MenuRecursivo/publicaciones_digitales/Est/Lib1584/libr o.pdf

La República (2019). Puno: Más casos de acoso y tocamientos en colegio de Juliaca. Recuperado de https://arepublica.pe/sociedad/2019/09/13/puno-mas-casos-de-acoso-ytocamientos-en-colegio-de-juliaca/

Morillo, B, Montero, L., y Colmenares, Z. (2012). Conocimiento de los padres en la prevención del abuso sexual infantil. Enfermería Global, 11(25), 1-

7. https://dx.doi.org/10.4321/S1695-61412012000100001

Organización Mundial de la Salud (2016). Comprender y abordar la violencia contra las mujeres: Violencia sexual. Recuperado de https://apps.who.int/iris/bitstream/handle/10665/98821/WHO_RHR_12.37_spa.pdf;jsessi onid=BE913C6E186442DE094C4F38290E3234 ? sequence $=1$

Quiroz, S. (2015). Implementación de un programa educativo para prevención del abuso sexual. Cajamarca 2015 (Tesis de maestría). Universidad Nacional Mayor de San Marcos, Lima, Perú. Recuperado de http://cybertesis.unmsm.edu.pe/handle/cybertesis/7937 
Redondo, S., Pérez, M., y Martínes, M. (2014). El riesgo de reincidencia en agresores sexuales. Papeles del psicólogo, 28(3), 187-195. Recuperado de http://www.redalyc.org/pdf/778/77828305.pdf

Rodríguez, Y., Aguilar, B., y García, I. (2012). Consecuencias psicológicas del abuso sexual infantil. Eureka, 9(1), 58-68. Recuperado de http://pepsic.bvsalud.org/pdf/eureka/v9n1/a07.pdf

Sánchez, J.E. (2015). Actitudes hacia la prevención del abuso sexual en estudiantes de primaria en una institución educativa en el año 2015 (Tesis de licenciatura), Universidad Nacional Mayor de San Marcos, Lima, Perú. Recuperado de https://cybertesis.unmsm.edu.pe/bitstream/handle/cybertesis/5997/Sanchez_gj.pdf?seque nce $=1$

Velázquez, M., Delgadillo, L.G., y González, L. (2013). Abuso sexual infantil. Técnicas básicas para su atención. Reflexiones, 92(1), 131-139. Recuperado de https://www.redalyc.org/pdf/729/72927050010.pdf

Vicente, M. (2017). Detectando el abuso sexual infantil. Pediatría atención primaria, 19(26), 39-47. Recuperado de http://scielo.isciii.es/scielo.php?script=sci_arttext\&pid=S1139$76322017000300005 \& \operatorname{lng}=\mathrm{es} \& \ln \mathrm{ln}=\mathrm{es}$.

Viñas, B. M., Mejía, M. A., Martínez, Y., y Islas, J. Y. (2015). Sexualidad Positiva y prevención de abuso sexual infantil en la frontera norte de México. PSIENCIA 7(1), 9-16. Recuperado de https $/ / w w w . r e d a l y c . o r g / p d f / 3331 / 333139283002 . p d f$ 